\title{
Review of: "Reprogrammable plasmonic topological insulators with ultrafast control"
}

\author{
Hong Liu ${ }^{1}$ \\ 1 Shandong University
}

Potential competing interests: The author(s) declared that no potential competing interests exist.

This study theoretically proposes and experimentally demonstrates a reprogrammable plasmonic topological insulator, where the topological propagation route can be dynamically changed at nanosecondlevel switching time, leading to an experimental demonstration of ultrafast multi-channel optical analogdigital converter.

To achieve the electrically driven programmability, each metallic element has six arms whose electromagnetic response can be electrically controlled via a positive-intrinsic-negative (PIN) diode. By controlling the switching state of the diode, the spatial symmetry of the unit cell can be controlled, and then the topological band can be the dynamically operated. The breakthrough of this work focus on the following aspects:

1. The $\mathrm{C}_{6}$ symmetry of the reprogrammable unit cell is a novel design, leads to the emergence of valleyHall states within the bandgap, which could inspire renewed research efforts in programmable valleytronics.

2. Each unit cell of the reprogrammable plasmonic topological insulator can be encoded by dynamically controlling its digital plasmonic states while keeping its geometry and material parameters unchanged, which has great potential for practical engineering application.

3. Each unit cell of the field-programmable topological electromagnetic metasurface realizes the function of independent electronic control coding, so the dynamic control precision and speed are unmatched by traditional reconfigurable topological photonic insulators.

4. The reprogrammable plasmonic topological insulator is fabricated by a widely-used printed circuit board (PCB) technology, and thus it can be seamlessly integrated with the commonly used PCB-based photoelectric integrated circuits.

The work brings the current studies of photonic topological insulators to a digital and intelligent era, which could open an avenue towards the development of software-defined photoelectric elements in high-speed communications and computation-based intelligent devices with built-in topological protection. 